\title{
Resection of multifocal non-small cell lung cancer when the bronchioloalveolar subtype is involved
}

\author{
Peter F. Roberts, MDa \\ Michaela Straznicka, MD \\ Primo N. Lara, $\mathrm{MD}^{\mathrm{b}}$ \\ Derrick H. Lau, MD \\ David M. Follette, MD ${ }^{a}$ \\ David R. Gandara, MD ${ }^{\mathrm{b}}$ \\ John R. Benfield, MD
}

Objective: Bronchioloalveolar lung cancer is commonly multifocal and can also present with other non-small cell types. The staging and treatment of multifocal non-small cell cancer are controversial. We evaluated the current staging of multifocal bronchioloalveolar carcinoma and the therapeutic effectiveness of resection when this tumor type is involved.

Methods: We reviewed our experience between 1992 and 2000 with complete pulmonary resections for bronchioloalveolar carcinoma. Kaplan-Meier survival curves were calculated from the dates of pulmonary resection.

Results: Among 73 patients with bronchioloalveolar carcinoma, 14 patients, 7 male and 7 female with a mean age of 65 years (51-87 years), had multifocal lesions without lymph node metastases. Follow-up was $100 \%$ for a median of 5 years (range 2.6-8.5 years). Tumor distribution was unilateral in 9 patients and bilateral in 5 patients. The multifocal nature of the disease was discovered intraoperatively in 4 patients. Nine patients had 2 lesions, 4 patients had 3 lesions, and 1 patient had innumerable discrete foci in a single lobe. Operative mortality was 0 . Postoperatively, 10 patients were staged pIIIB or pIV on the basis of multiple foci of similar morphology; 4 patients had some differences in histology (implying multiple stage 1 primaries). The median survival time to death from cancer was 14 months (141 days-5.6 years). The overall 5-year survival after resection of multifocal bronchioloalveolar carcinoma was $64 \%$. Unilateral or bilateral distribution had no impact on survival.

Conclusions. The current staging system is not prognostic for multifocal bronchioloalveolar carcinoma without lymph node metastases. Complete resection of multifocal non-small cell lung cancer when bronchioloalveolar carcinoma is a component may achieve survivals similar to that of stage I and II unifocal non-small cell lung cancer. When bronchioloalveolar carcinoma is believed to be one of the cell types in multifocal disease without lymph node metastases, consideration should be given to surgical resection.

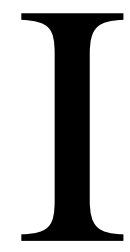

n clinical practice, the many types and subtypes of non-small cell lung cancer (NSCLC) have been treated as a single entity. The evaluation, staging, and treatment of NSCLC have been fairly standardized. Bronchioloalveolar carcinoma (BAC) of the lung is a subtype of adenocarcinoma that has enough distinct clinical features to justify special consideration when offering a prognosis. With the rising incidence of $\mathrm{BAC},{ }^{1}$ thoracic surgeons will be faced with increasing preoperative diagnoses of BAC, thus allowing time to formulate a treatment plan tailored for this specific lesion. 
BAC has a lepidic growth pattern along alveolar walls. Computed tomography (CT) typically shows either a nodular type or diffuse infiltrative type of growth pattern. Malasse ${ }^{2}$ described the diffuse type in 1876 and associated it with a poor prognosis. In 1960, Liebow ${ }^{3}$ noted the association of the small peripheral airways and alveolar spaces and introduced the term "bronchioloalveolar carcinoma." BAC has been further subdivided into mucinous and nonmucinous types. BAC may present as a multifocal process; the reported incidence is as high as $25 \%{ }^{1}$ Multiple tumor types can exist in a synchronous fashion with BAC.

The staging and treatment of multifocal lung cancer have been controversial, at least in part because it can be hard to differentiate multiple sites of primary cancer from intrapulmonary metastases (IPMs). ${ }^{4}$ Related to this issue are 2 alterations in the staging system of lung cancer that have occurred in the last decade. In 1992, the Union Internationale Contre le Cancer and the American Joint Committee on Cancer revised the TNM classification for lung cancer and classified IPMs as local progression rather than distant metastases. ${ }^{4,5}$ In 1997, another change was introduced: IPMs were to be designated as T4 lesions when they occurred in the same lobe as the primary tumor, therefore placing patients with IPMs in the same lobe in stage IIIB. ${ }^{6}$ IPMs in all other lobes were designated as distant metastases, M1, and therefore stage IV disease. At present there is no standardized technique of determining monoclonality.

What outcome might one expect after complete resection of multifocal NSCLC without lymph node metastases (N0)? Two recently published studies attempted to further clarify this issue. Batafarano and colleagues ${ }^{7}$ have reported encouraging results after complete surgical resection of multifocal NSCLC, but their data regarding BAC and combinations of tumor types was limited. Ebright and colleagues ${ }^{8}$ reviewed their experience with multifocal BAC and concluded that even patients in the advanced stage should undergo resection. They included only BAC pathology. We reviewed our experience to gain further insight on a limited body of literature regarding the adequacy of the current staging system to predict survival in multifocal BAC. Furthermore, there are increasing data to support the belief that BAC has an independently favorable prognosis when compared with other NSCLC. ${ }^{8}$ Therefore, one would expect survival to be higher in a subgroup of patients with multifocal disease when BAC is involved.

\section{Methods}

The study was performed retrospectively on patients who underwent resection at a single institution. Our surgical pathology database of all resected lung specimens was searched from 1992 to 2000 for all patients whose final diagnoses included BAC or adenocarcinoma with foci of BAC.

BAC was diagnosed according to the recent World Health Organization criteria ${ }^{9}$ when a bronchioloalveolar growth pattern occurred without stromal, vascular, or pleural invasion. Patients with multifocal disease were identified and underwent a more detailed chart review. Multifocal disease was defined as 2 or more foci of tumor separated by normal lung tissue. Tumors that differed histologically were designated as multiple primary tumors. When tumors were histologically similar, they were designated as IPMs. Only patients who underwent a complete resection with curative intent were included in the study. A thorough review of the medical records was performed on these patients, and pathologic diagnosis was again confirmed in this group. Patients with a preoperative diagnosis of $\mathrm{N} 2$ disease received induction therapy and therefore were not included in the study. In addition, patients who underwent operations with the intent of only diagnosis (biopsies) and patients who were discovered to have a positive margin were not included.

Preoperative workup in all patients included chest CT scanning from lung apex to adrenal glands. Complete mediastinal lymph node sampling was performed at all operations. Patients were staged according to the current TNM classification. ${ }^{6}$ Follow-up was achieved with chart reviews and telephone contact when the most recent office visit was more than 6 months before the review. Dates of death were established through family members and the Social Security Death Index.

Survival was calculated by the Kaplan-Meier estimation using the date of initial pulmonary resection and date of death or last contact as the beginning and end points. Differences in survival were calculated using log-rank analysis.

\section{Results}

Between 1992 and 2000, 105 patients with BAC were found. Thirty-two of these underwent only biopsies or incomplete resections and were therefore excluded from the study. Seventy-three patients underwent complete pulmonary resections for at least 1 focus of BAC.

Fourteen patients, 7 men and 7 women with a mean age of 65 years (range 51-87 years), were identified who had N0 multifocal disease (T1-T4). Nine patients had unilateral BAC, and 5 patients had bilateral lesions. Follow-up in the study was $100 \%$. Median duration of follow-up of the 8 currently living patients was 5 years (range 2.6-8.5 years). The 14 patients are summarized in Figure 1.

\section{Preoperative Diagnosis}

In 10 cases, the multifocal nature of the disease was known preoperatively. In 4 cases, the multifocal nature of the disease was discovered intraoperatively and postoperatively. Three of these cases occurred early in the study when CT scanners with comparatively low resolution were being used.

\section{Operations}

Fourteen patients had 30 pulmonary resections in 18 operations including 7 unilateral thoracotomies, 2 video-assisted resections, 2 median sternotomies (each requiring a subsequent thoracotomy), 2 staged bilateral thoracotomies, and 1 
same-day bilateral thoracotomy. There were 12 lobectomies, 15 wedge resections, and 3 formal segmentectomies.

Among the 5 patients with bilateral BAC, 2 underwent sternotomies, including 1 patient who underwent a complete resection with a segmentectomy and 2 wedge resections. The second patient underwent a right upper lobectomy through a sternotomy, but the planned left lower lobectomy could not be safely and completely resected through that incision. Accordingly, left lower lobectomy was performed 2 months later. Three patients underwent bilateral thoracotomies, including 1 single operation and 2 staged procedures at 2- and 3-month intervals.

There were no operative deaths. Two serious complications (14\%) included a deep venous thrombosis and pulmonary embolism in 1 patient and an empyema in 1 patient. Both patients recovered fully. The single minor complication was a delayed pneumothorax after 3 wedge resections.

\section{Pathologic Findings}

Nine patients had 2 foci of cancer, 4 patients had 3 foci, and 1 patient had many discrete foci of BAC within a single lobe. The 14 patients were staged in the following way. Two patients were staged IIIB on the basis of multiple lesions within the same lobe. Eight patients had stage IV disease on the basis of foci of BAC in multiple lobes. Two patients had clearly different pathology in the second foci, a neuroendocrine tumor in 1, and a poorly differentiated large cell tumor in the other, which were therefore multiple stage I carcinomas. The final 2 patients had a combination of adenocarcinoma with BAC features in 1 tumor and pure $\mathrm{BAC}$ in the other lesion(s). These patients were staged with independent tumors. No patients had mediastinal lymph node involvement.

Two patients had a history of lung cancers, both approximately 2 years before resection of $\mathrm{N} 0$ multifocal BAC. The first patient was a 70-year-old woman who had been treated for a limited small cell cancer with wedge resection and postoperative chemotherapy. Her multifocal disease occurred 2 years later and was found at resection to have both a focus of BAC and a neuroendocrine tumor; both were completely resected. She remains disease-free at 2.8 years follow-up. The second patient was a 69 -year-old man who had undergone resection for an adenocarcinoma that was histologically different from his subsequent cancers. When his multifocal BAC presented 2 years later, he underwent a completion right upper lobectomy. He developed tumor recurrence initially confined to the thorax 3 years later and elected not to undergo treatment. He has had slowly progressing disease for 2 years.

A 68-year-old woman also had developed a recurrence 4.6 years after her original sternotomy for bilateral BAC. She elected to undergo re-resection for her new foci of BAC. She tolerated this well, but when it recurred 2 years

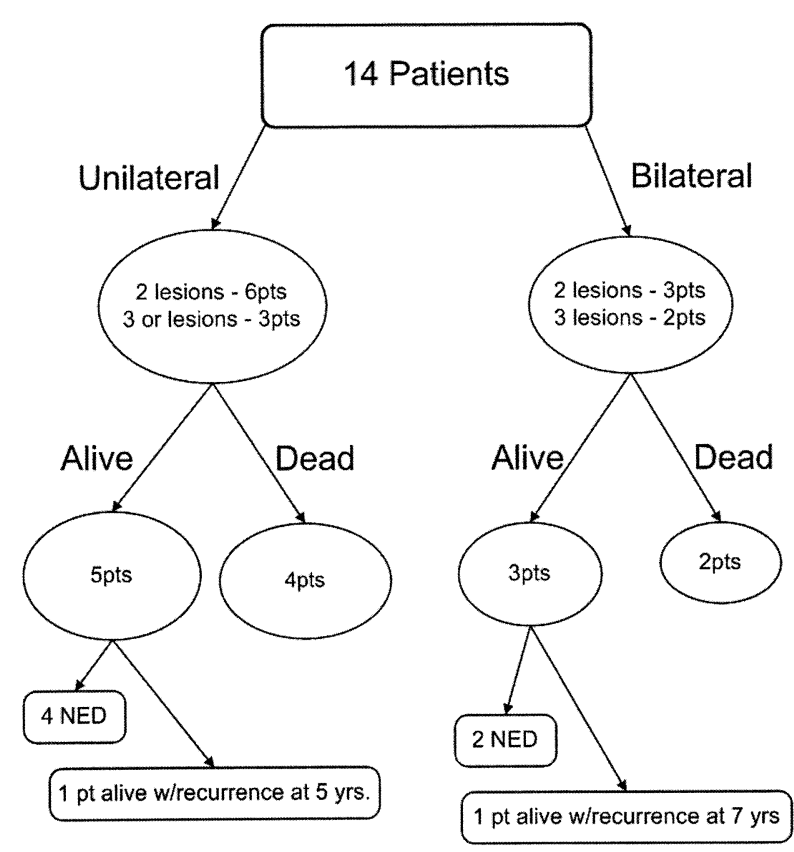

Figure 1. Outcome of the 14 patients with unilateral and bilateral distribution of their tumors. NED, No evidence of disease.

later she opted not to receive any further treatment. She remains active 6.7 years after her initial resection without any evidence of extrathoracic disease. It is interesting that she had been followed for 2 years before her initial resection for what was her slowly increasing BAC. This patient illustrates the often insidious nature of some types of BAC.

Five patients died of tumor recurrence. Two patients with non-BAC histology had extrathoracic spread before their death. Their median survival was 14 months (range 141 days-5.6 years). The patient who died 5 months postoperatively had a very aggressive form of BAC. No patient with lethal tumor recurrence underwent chemotherapy or radiation. The final death in the group was a patient who died as a result of pancreatic cancer 1 year after the pulmonary resection.

The overall survival in the study group was $64 \%$ at 5 years (Figure 2). Currently, 6 patients are alive and well without known recurrence of BAC or any other type of lung cancer. Two of the surviving 8 patients have known tumor recurrence and are alive at more than 5 years after their initial resection. There was no difference in survival related to the number of or distribution of lesions.

\section{Discussion}

Most patients with BAC in our study were designated with advanced disease because they were in stage IIIB or stage IV according to current staging nomenclature. Four patients had multiple primary tumors. The expected 5-year survival for NSCLC in stages IIIB and IV is approximately 5\% and 


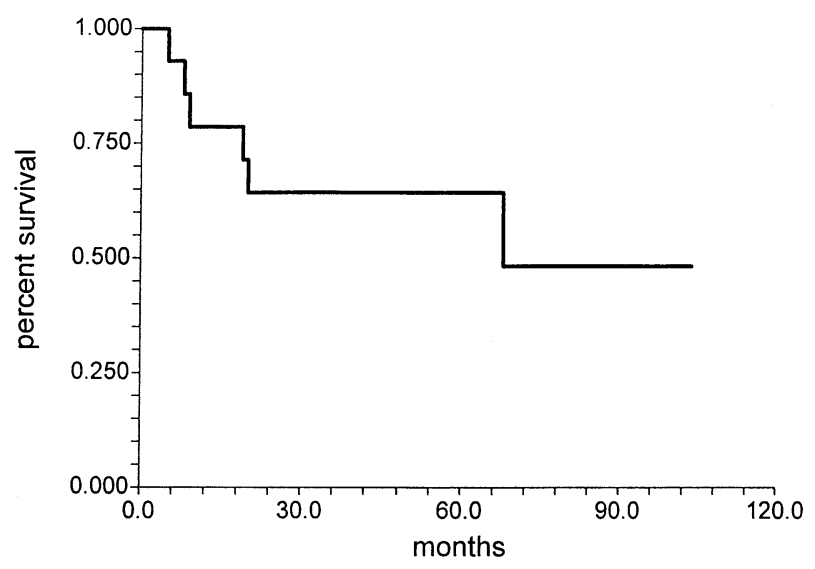

Figure 2. Overall survival of 14 patients with resected multifocal bronchoalveolar lung cancer. Survival was calculated from initial pulmonary resection until death or last follow-up. At 5 years, overall survival was $64 \%$.

less than $1 \%$, respectively. ${ }^{10}$ The survival of $64 \%$ in our study greatly exceeds this and is much greater than reports for survival in non-BAC multifocal disease. Vansteenkiste and coworkers ${ }^{11}$ reported a 5 -year survival of $33 \%$ when all cell types were considered. This indicates a serious deficiency in applying the current staging system to multifocal disease if BAC is involved. As Ebright and colleagues ${ }^{8}$ claimed, this does not seem to depend on the BAC subtype.

Evaluating the validity of staging systems is important because staging criteria evolve as a result of compiled experience. After the 1997 revisions, Okada and associates $^{12}$ evaluated 89 patients who had ipsilateral IPMs. All patients by definition were either in stage IIIB or stage IV. By using the 1997 system, they compared patients with stage III and IV disease with and without IPMs. Patients with stage IV disease on the basis of metastases to a lung lobe other than the one that had contained the primary metastasis had significantly better survival than patients who were in stage IV because of other sites of metastasis. Within the IIIB group, there were significant survival differences between the patients with N0, N1, and N2 disease. In other words, Okada and coworkers confirmed that lymph node status ( $\mathrm{N}$ factor) was a better predictor of survival than the number or location of IPMs that could be surgically resected. Their findings, like ours, indicated that patients with multifocal disease without mediastinal involvement have a better prognosis than a patient with $\mathrm{N} 2$ or N3 disease. Okada and associates also concluded that the 1997 revisions were less acceptable than the 1992 revisions with regard to the significance of IPM. These investigators, however, intentionally avoided including BAC in their study because of their multicentricity and unique behavior.

When Nakajima and colleagues ${ }^{13}$ reported their experience with IPM, they included patients with BAC. They reviewed 50 patients with NSCLC who had IPMs, including 29 patients with well-differentiated adenocarcinoma with bronchoalveolar spread. Patients without vascular or lymphatic invasion and with $\mathrm{T}$ status less than or equal to $2 \mathrm{had}$ significantly better survival than those who had vascular or lymphatic invasion or $\mathrm{T}$ status greater than 2 . Fifteen patients had BAC without vascular or lymphatic spread with a $\mathrm{T}$ status less than or equal to 2; their actuarial 5-year survival was $100 \%$ with a mean follow-up of 28 months. Nakajima and coworkers, however, cautioned that the exceptionally high survival in their study may have been the result of some erroneous diagnoses of BAC when atypical adenomatous hyperplasia was actually present. Accordingly, in our study we reviewed the pathology slides to confirm the diagnosis of BAC in accordance with new World Health Organization guidelines. The question of multiple primary tumors (localized cancer in several places) versus true metastasis (systemic disease) is central to understanding BAC. Barsky and colleagues ${ }^{1}$ in 1994 published their genetic research with BAC using a novel strategy for clonality determination. They determined that tumors in these locations were mostly multiclonal independent cancers. They further suggested that because of this fact, lung-sparing operations (wedge resections) should be performed. A subsequent genetic study argued for a monoclonal origin. ${ }^{14}$ This study involved larger numbers of patients, and because the genetic investigators believed their data supported a truly metastatic hypothesis, they questioned the role of surgery in patients with multifocal disease. This issue has not been evaluated in a large clinical series, and we made no attempt to do so here. Although it is an important biologic question, it may not be immediately clinically relevant because the overall survival in our patients in other series ${ }^{8}$ indicates that multifocal BACs should be resected regardless of their clonal origin.

The wide variety of clinical behaviors within BAC has complicated the issue. Groups have reviewed their patients in an attempt to improve the ability to predict outcome and tailor treatment on the basis of the histologic subtype and radiographic appearance of tumors. ${ }^{15}$ Ebright and colleagues ${ }^{8}$ have subsequently suggested that histologic subtype does not affect prognosis whereas stage and clinical patterns do. Holst and colleagues ${ }^{14}$ noted that as patients age they are less likely to have metastases. Dumont and associates ${ }^{16}$ reported on a group of patients who had had stable parenchymal lesions for 2 to 7 years (mean 4 years) that were ultimately determined to be BACs. They had received no treatment. These facts may indicate that elderly patients with slow-growing lesions may be best treated with close observation. Perhaps it is the tumor biology and not the surgery that is accounting for survival. This is underscored by 2 of our patients who have experienced long-term survival with known recurrences. Clearly some types of 
BAC behave much differently than other NSCLCs. Observation of resectable lung cancer would require a new treatment paradigm based on a tumor's specific predicted behavior. Retrospective reviews may not be able to validate this idea, and at the present time randomized trials do not exist.

Conventional chemotherapy has had poor results with BAC, but newer drugs such as epidermal growth factor receptor-tyrosine kinase inhibitors, "Iressa," and vaccine therapies have shown some initial promise. ${ }^{17}$ Studies evaluating the role of these drugs specifically in BAC are ongoing. Perhaps these drugs will improve survival in both early and advanced stages of BAC. Unanswered questions and different opinions reflect the complexities and heterogeneity of this tumor subtype.

Confusion would result if modifications were made to the lung cancer staging system too frequently. This would translate into difficulty in patient care, clinical trial development and interpretation, and comparisons of data among institutions. Thus, even though we believe that the current staging system is inadequate for multifocal NO BAC, we continue to use it. However, we support previous reports that indicate a need for ongoing evaluation with eventual modifications to the staging system based on the collection of more data. We hope that a greater understanding of individual tumor biology will add to the clarity and accuracy of new staging systems that may take into account tumor type.

On the basis of our experience, we continue to evaluate multifocal disease when BAC is present with an eye toward resection. Although many of these patients will be clinically staged IIIB or IV, we recommend operation when complete resection can be safely achieved. Even in cases of repeat resections, long-term survival can be achieved.

We acknowledge Ronald Hayes, PA-C, and Shirley Cable for their great assistance in data collection and preparation of this article.

\section{References}

1. Barsky SH, Grossman DA, Ho J, Holmes EC. The multifocality of bronchioloalveolar lung carcinoma: evidence and implications of a multiclonal origin. Mod Pathol. 1994;7:633-40.

2. Malassez L. Examen histologique d'un cas de cancer enciphaloide du poumon (epithelioma). Arch Physiol Norm Pathol. 1876;3:353-72.

3. Liebow AA. Bronchiolo-alveolar carcinoma. Adv Intern Med. 1960; 10:329-58

4. American Joint Committee on Cancer. Manual for staging of cancer. 4th ed. Philadelphia: Lippincott; 1992.

5. Mountain CF, Libshitz HI, Hermes KE. Lung cancer handbook for staging and imaging. Houston: CF Mountain; 1992.

6. Mountain CF. Revisions in the International System for Staging Lung Cancer. Chest. 1997;111:1710-7.

7. Batafarano RJ, Meyers BF, Guthrie TJ, Cooper JD, Patterson GA. Surgical resection of multi-focal non-small cell lung cancer is associated with prolonged survival. Ann Thorac Surg. 2002;74:988-94.

8. Ebright MI, Zakowski MF, Martin J, Venkatraman ES, Miller VA, Bains MS, et al. Clinical pattern and pathologic stage but not histologic features predict outcome for bronchioloalveolar carcinoma. Ann Thorac Surg. 2002;74:1640-7.

9. Travis WD, Colby TV, Corrin B, Shimosato Y, Brambilla E, Sobin LH, et al. In: World Health Organization Pathology Panel, editor. World Health Organization International Histological Classification of Tumors. Berlin: Springer Verlag; 1995. p. 5.

10. Mountain CF. The international system for staging lung cancer. Semin Surg Oncol. 2000;18:106-15.

11. Vansteenkiste JF, De Belie B, Denefee GJ, Demedts MG, De Lyn PR, Van Raemdonck DE, et al. Practical approach to patients presenting with multiple synchronous suspect lung lesions: a reflection on the current TNM classification based on 54 cases with complete followup. Lung Cancer. 2001;34:169-75.

12. Okada M, Tsubota N, Yoshimura M, Miyamoto Y, Nakai R. Evaluation of TNM classification for lung carcinoma with ipsilateral intrapulmonary metastasis. Ann Thorac Surg. 1999;68:326-31.

13. Nakajima J, Furuse A, Oka T, Kohno T, Ohtsuka T. Excellent survival in a subgroup of patients with intrapulmonary metastasis of lung cancer. Ann Thorac Surg. 1996;61:158-62.

14. Holst VA, Finkelstein S, Yousem SA. Bronchioloalveolar adenocarcinoma of lung: monoclonal origin for multifocal disease. Am J Surg Pathol. 1998;22:1343-50

15. Caretta A, Cannetto B, Calori G, Ceresoli GL, Campagnoli E, Arrigoni $\mathrm{G}$, et al. Evaluation of radiological and pathological prognostic factors in surgically-treated patients with bronchoalveolar carcinoma. Eur J Cardiothorac Surg. 2001;20:367-71.

16. Dumont P, Gasser B, Rouge C, Massard G, Wihlm J-M. Bronchoalveolar carcinoma. Histopathologic study of evolution in a series of 105 surgically treated patients. Chest. 1998;113:391-5.

17. Fukuoka M, Yano S, Giaccone G, Tamura T, Nakagawa K, Douillard JY, et al. Multi-institutional randomized phase II trial of gefitinib for previously treated patients with advanced non-small-cell lung cancer. J Clin Oncol. 2003;21:2237-46.

\section{Discussion}

Dr Richard Whyte (Stanford, Calif). In this article, the authors present the outcomes of 14 patients with multifocal BAC of the lung. They suggest that a conventional staging system would assign these patients to either stage IIIb or IV depending on whether the lesions are in the same lobe or different lobes, and that such patients would have an anticipated survival of less than $10 \%$. The observed survival was greater than $60 \%$, and this clearly indicates one of the shortcomings of the conventional staging system. It is very helpful for us to know what happens to these patients in the long run.

Dr Roberts, I have 3 questions for you. First, your patients with multifocal disease seem to represent approximately $20 \%$ of the patients with BAC whom you have seen. What happened with the other $80 \%$ ? Were they followed in the long run for many years? If so, would you expect your $20 \%$ to actually be a significantly higher percentage of all the patients with BAC presenting with multifocal disease?

Dr Roberts. One deficiency of our study is that we did not review all of the pathology slides. Perhaps some of the other patients whom we have treated for multifocal disease in fact had $\mathrm{BAC}$, and it is true that what appears to be unifocal disease may some day be multifocal disease or recurrence. We did not review in detail all of our other patients with BAC, but the overall survival of those patients does seem to be about what you would expect with stage I NSCLC.

Dr Whyte. My second question relates to the nomenclature of BAC. In our weekly tumor board meetings, we frequently hear about adenocarcinoma with bronchoalveolar features versus BAC. 